\title{
Acknowledgement to BJD 2017 reviewers
}

\section{We are grateful to the following people who acted as reviewers for articles submitted to the British Journal of Diabetes during 2017.}

Dr Karen Adamson, St John's Hospital, Livingston

Dr John Campbell, University of Bath

Dr Pankaj Chaturvedi, Doncaster Bassetlaw Hospitals

Dr Kelly Cheer, Wythenshawe Hospital

Dr Louise Curtis, University Hospital, Southampton

Dr Srilatha Dampetla, Lancaster

Dr Umesh Dashora, Conquest Hospital, Hastings

Dr Ketan Dhatariya, Norfolk \& Norwich University Hospital

Dr Mark Evans, Institute of Metabolic Science, Cambridge

Professor Miles Fisher, Glasgow Royal Infirmary

Professor Roger Gadsby, Warwick Medical School

Dr Alison Gallagher, Universitry Hospitals of Leicester

Dr Jyothis George, Churchill Hopspital, Oxford

Dr Susana Gonzalez-Sena, Bradford Royal Infirmary

Professor Stephen Gough, Churchill Hospital, Oxford

Dr Peter Hammond, Harrogate District Hospital

Professor Hugh Jones, Barnsley Hospital NHSFT

Professor Edward Jude, Tameside Hospital

Dr Muhammed Karamat, Heart of England NHSFT

Dr Ruth Krone, Birmingham Children's Hospital
Dr John Lindsay, Mater Hospital, Belfast

Dr Andrew Macklin, Dorset County Hospital, Dorchester

Dr Susan Manley, Birmingham University Hospitals

Dr Kamrudeen Mohammed, Hull Royal Infirmary

Dr Neil Munro, Chelsea \& Westminster Hospital, London

Dr Dinesh Nagi, Pinderfields General Hospital, Wakefield

Dr Adam Nicholls, University Hospital, Southampton

Dr Dipesh Patel, Royal Free Hospital, London

Dr Raghavan Rajeev, New Cross Hospital, Wolverhampton

Dr Susannah Rowles, Fairfield Hospital, Bury

Dr Ricky Saharia, Hull Royal Infirmary

Dr Sofia Salahuddin, University Hospital, Birmingham

Professor Alan Sinclair, Luton \& Dunstable NHSFT

Professor Baldev Singh, New Cross Hospital, Wolverhampton

Dr Karen Tait, Birmingham Community Healthcare NHS Trust

Dr Jennifer Tringham, Frimley Park Hospital

Dr lan Wallace, Royal Victoria Hospital, Belfast

Dr Chris Walton, Hull Royal Infirmary

Dr Ken Yan Thong, Perth, Western Australia 